\title{
EXTENDING GENERALIZED FIBONACCI SEQUENCES AND THEIR BINET-TYPE FORMULA
}

\author{
MUSTAPHA RACHIDI AND OSAMU SAEKI
}

Received 10 March 2006; Accepted 2 July 2006

We study the extension problem of a given sequence defined by a finite order recurrence to a sequence defined by an infinite order recurrence with periodic coefficient sequence. We also study infinite order recurrence relations in a strong sense and give a complete answer to the extension problem. We also obtain a Binet-type formula, answering several open questions about these sequences and their characteristic power series.

Copyright (c) 2006 M. Rachidi and O. Saeki. This is an open access article distributed under the Creative Commons Attribution License, which permits unrestricted use, distribution, and reproduction in any medium, provided the original work is properly cited.

\section{Introduction}

The notion of an $\infty$-generalized Fibonacci sequence ( $\infty$-GFS) has been introduced in [7] and studied in $[1,8,10]$. This class of sequences defined by linear recurrences of infinite order is an extension of the class of ordinary (weighted) $r$-generalized Fibonacci sequences ( $r$-GFSs) with $r$ finite defined by linear recurrences of $r$ th order (e.g., see [3-6, 9], etc.). Such sequences are defined as follows. Let $\left\{a_{i}\right\}_{i=0}^{\infty}$ and $\left\{\alpha_{-i}\right\}_{i=0}^{\infty}$ be two sequences of complex numbers, where $a_{i} \neq 0$ for some $i$. The associated $\infty$-GFS $\left\{V_{n}\right\}_{n \in \mathbb{Z}}$ is defined by

$$
\begin{gathered}
V_{n}=\alpha_{n} \quad \text { if } n \leq 0, \\
V_{n}=\sum_{i=0}^{\infty} a_{i} V_{n-i-1} \quad \text { if } n \geq 1 .
\end{gathered}
$$

The sequences $\left\{a_{i}\right\}_{i=0}^{\infty}$ and $\left\{\alpha_{-i}\right\}_{i=0}^{\infty}$ are called the coefficient sequence and the initial sequence, respectively. As is easily observed, the general terms $V_{n}$ may not necessarily exist. In [1], necessary and sufficient conditions for the existence of the general terms have been studied. When there exists an $r \geq 1$ such that $a_{i}=0$ for all $i \geq r$, we call the sequence $\left\{V_{n}\right\}_{n \geq-r+1}$ an $r$-GFS with initial sequence $\left\{V_{-r+1}, V_{-r+2}, \ldots, V_{0}\right\}$. For an $r$-GFS, 
the numbering often starts with $V_{1}$ instead of $V_{-r+1}$. In such a case, all the numberings shift by $r$.

The case where the coefficient sequence $\left\{a_{i}\right\}_{i=0}^{\infty}$ is periodic, that is, the case where there exists an $r \geq 1$ such that $a_{i+r}=a_{i}$ for every $i \geq 0$ is considered in [2]. It was shown that in such a case, the associated $\infty$-GFS is an $r$-GFS associated with the coefficient sequence

$$
\left\{a_{0}, a_{1}, \ldots, a_{r-2}, a_{r-1}+1\right\}
$$

and the initial sequence $\left\{V_{1}, V_{2}, \ldots, V_{r}\right\}$, where $r \geq 1$, is the period. Thus, the following problem naturally arises. Given an $r$-GFS, can one always extend it to an $\infty$-GFS associated with a periodic coefficient sequence? If it is not always the case, then characterize those r-GFSs which can be extended to an $\infty$-GFS associated with a periodic coefficient sequence.

In this paper, we first show that under a mild condition on the coefficients, an $r$ GFS can always be extended to an $\infty$-GFS associated with a periodic coefficient sequence (Proposition 2.1).

On the other hand, it was shown that a root of the characteristic polynomial of an $r$-GFS does not always give an $\infty$-GFS associated with a periodic coefficient sequence (see [2, Example 3.4]). In order to analyze this type of phenomena, in Section 3, we introduce the notion of a strongly $\infty$-GFS, imposing the condition (1.2) not only for $n \geq 1$, but for all $n \in \mathbb{Z}$. In a sense, this condition is more natural than requiring the equation only for $n \geq 1$, and it has already appeared in [7, Problem 3.11]. The main result of this paper is a characterization theorem of those $r$-GFSs which can be extended to a strongly $\infty$ GFS associated with a periodic coefficient sequence (Theorem 3.2). This gives a complete solution to the problem mentioned above in the case of strongly $\infty$-GFSs. The characterization will be given in terms of the zeros of the characteristic polynomial.

As a corollary, we will give a Binet-type formula for such sequences in terms of the roots of the associated characteristic polynomial (Corollary 3.7). The characteristic polynomial of an $r$-GFS is closely related to the characteristic power series of the associated periodic coefficient sequence (see Remark 3.4), and we will see that this Binet-type formula uses only those zeros of the characteristic power series inside the circle of convergence. This gives a positive answer to [7, Problem 4.5] in the case of periodic strongly $\infty$-GFSs. We will also see that our characterization theorem gives a complete solution to [7, Problem 3.11] in the case where the coefficient sequence is periodic (Corollary 3.8).

\section{Extending an $r$-GFS to a periodic $\infty$-GFS}

Let $\left\{a_{i}\right\}_{i=0}^{\infty}$ be a coefficient sequence. If there exists a positive integer $r$ such that

$$
a_{i+r}=a_{i} \quad \forall i \geq 0
$$

then we call the associated sequence $\left\{V_{n}\right\}_{n \in \mathbb{Z}}$ defined by (1.1) and (1.2) a periodic $\infty$-generalized Fibonacci sequence. It was shown that in such a case, the subsequence $\left\{V_{n}\right\}_{n=1}^{\infty}$ is an $r$-GFS associated with the coefficient sequence (1.3) and the initial sequence $\left\{V_{1}, V_{2}, \ldots, V_{r}\right\}$ (see [2]).

Conversely, suppose that an $r$-GFS $\left\{V_{n}\right\}_{n=1}^{\infty}$ associated with the coefficient sequence (1.3) is given. We would like to determine whether it can be extended to a periodic $\infty$-GFS 
associated with the periodic coefficient sequence

$$
\left\{a_{0}, a_{1}, \ldots, a_{r-1}, a_{0}, a_{1}, \ldots, a_{r-1}, \ldots\right\}
$$

or not.

Set

$$
T(x)=\sum_{i=0}^{r-1} a_{i} x^{r-1-i}
$$

Then we have the following.

Proposition 2.1. Let $\left\{V_{n}\right\}_{n=1}^{\infty}$ be an $r$-GFS associated with the coefficient sequence (1.3). If $T(x)$ does not have any root $\xi \in \mathbb{C}$ with $\xi^{r}=1$, then there exists a sequence $\left\{V_{-n}\right\}_{n=0}^{\infty}$ such that $\left\{V_{n}\right\}_{n \in \mathbb{Z}}$ is an $\infty$-GFS associated with the periodic coefficient sequence (2.2).

Proof. In the following, we set $a_{k}=a_{k^{\prime}}$ for $k \geq r$, where $k^{\prime} \equiv k(\bmod r)$ and $0 \leq k^{\prime} \leq$ $r-1$.

Let us consider the following set of $r$ linear equations with respect to the variables $\alpha_{0}, \alpha_{-1}, \ldots, \alpha_{-r+1}$ :

$$
\begin{gathered}
V_{1}=a_{0} \alpha_{0}+a_{1} \alpha_{-1}+\cdots+a_{r-1} \alpha_{-r+1}, \\
V_{2}=a_{0} V_{1}+a_{1} \alpha_{0}+a_{2} \alpha_{-1}+\cdots+a_{r-1} \alpha_{-r+2}+a_{0} \alpha_{-r+1}, \\
V_{3}=a_{0} V_{2}+a_{1} V_{1}+a_{2} \alpha_{0}+a_{3} \alpha_{-1}+\cdots+a_{r-1} \alpha_{-r+3}+a_{0} \alpha_{-r+2}+a_{1} \alpha_{-r+1}, \\
\vdots \\
V_{r}=a_{0} V_{r-1}+a_{1} V_{r-2}+\cdots+a_{r-2} V_{1}+a_{r-1} \alpha_{0}+a_{0} \alpha_{-1}+a_{1} \alpha_{-2}+\cdots+a_{r-2} \alpha_{-r+1} .
\end{gathered}
$$

Set $I_{i}=\left(a_{i-1}, a_{i}, \ldots, a_{r+i-2}\right)$ for $i=1,2, \ldots, r-1$. Furthermore, define $I_{i}^{\prime}$ inductively by $I_{1}=$ $I_{1}^{\prime}$ and

$$
I_{i}^{\prime}=a_{0} I_{i-1}^{\prime}+a_{1} I_{i-2}^{\prime}+\cdots+a_{i-2} I_{1}^{\prime}+I_{i}
$$

for $i \geq 2$. Then the above set of $r$ equations can be written as

$$
\left(\begin{array}{c}
V_{1} \\
V_{2} \\
\vdots \\
V_{r}
\end{array}\right)=\left(\begin{array}{c}
I_{1}^{\prime} \\
I_{2}^{\prime} \\
\vdots \\
I_{r}^{\prime}
\end{array}\right)\left(\begin{array}{c}
\alpha_{0} \\
\alpha_{-1} \\
\vdots \\
\alpha_{-r+1}
\end{array}\right) .
$$

Since the $r \times r$ matrices

$$
\left(\begin{array}{c}
I_{1}^{\prime} \\
I_{2}^{\prime} \\
\vdots \\
I_{r}^{\prime}
\end{array}\right), \quad A=\left(\begin{array}{c}
I_{1} \\
I_{2} \\
\vdots \\
I_{r}
\end{array}\right)
$$

have the same determinants, the above set of $r$ equations has a solution if $\operatorname{det} A \neq 0$. 


\section{Extending generalized Fibonacci sequences}

On the other hand, by our assumption on $T(x)$, we have $\operatorname{det} A \neq 0$ (for details, see [2, the proof of Proposition 2.2]). Therefore, there exist $\alpha_{0}, \alpha_{-1}, \ldots, \alpha_{-r+1}$ which satisfy the above set of $r$ linear equations.

Set $V_{n}=\alpha_{n}$ for $n=0,-1, \ldots,-r+1$ and $V_{n}=0$ for $n<-r+1$. Let us show that the sequence $\left\{V_{n}\right\}_{n \in \mathbb{Z}}$ thus defined is an $\infty$-GFS associated with the coefficient sequence (2.2).

Since $\alpha_{0}, \alpha_{-1}, \ldots, \alpha_{-r+1}$ satisfy the above $r$ equations, we see that (1.2) is valid for $n=$ $1,2, \ldots, r$. Suppose by induction that $(1.2)$ is valid for $n=1,2, \ldots, k$ with $k \geq r$. Since $\left\{V_{n}\right\}_{n=1}^{\infty}$ is an $r$-GFS associated with the coefficient sequence (1.3), we have

$$
\begin{aligned}
V_{k+1}= & a_{0} V_{k}+a_{1} V_{k-1}+\cdots+a_{r-2} V_{k-r+2}+\left(a_{r-1}+1\right) V_{k-r+1} \\
= & a_{0} V_{k}+a_{1} V_{k-1}+\cdots+a_{r-2} V_{k-r+2}+a_{r-1} V_{k-r+1} \\
& +\left(a_{0} V_{k-r}+a_{1} V_{k-r-1}+\cdots\right) \\
= & \sum_{i=0}^{\infty} a_{i} V_{k-i} .
\end{aligned}
$$

This shows that (1.2) is valid for $n=k+1$ as well. Hence the sequence $\left\{V_{n}\right\}_{n \in \mathbb{Z}}$ is a periodic $\infty$-GFS associated with the coefficient sequence (2.2).

Remark 2.2. As the above proof shows, if the vector ${ }^{t}\left(V_{1}, V_{2}, \ldots, V_{r}\right)$ belongs to the vector space spanned by the $r$ vectors ${ }^{t} I_{1},{ }^{t} I_{2}, \ldots,{ }^{t} I_{r}$, then we have the same conclusion without the assumption on $T(x)$.

Remark 2.3. In the above proposition, we have constructed an extension $\left\{V_{n}\right\}_{n \in \mathbb{Z}}$ such that $V_{n}=0$ for all $n<-r+1$. If we impose this condition, then the extension is unique as the above proof shows. However, an extension is not unique in general. For example, for $\alpha_{0}, \alpha_{-1}, \ldots, \alpha_{-r+1}$ as constructed in the proof, and for arbitrary $\beta_{0}, \beta_{-1}, \ldots, \beta_{-r+1}$, define $\left\{V_{-n}\right\}_{n=0}^{\infty}$ by

$$
V_{n}= \begin{cases}\beta_{n}, & 0 \geq n \geq-r+1, \\ \alpha_{n+r}-\beta_{n+r}, & -r \geq n \geq-2 r+1, \\ 0, & n \leq-2 r .\end{cases}
$$

Then it is easy to see that $\left\{V_{n}\right\}_{n \in \mathbb{Z}}$ is also a required extension.

Example 2.4. As in [2, Example 3.4], let us consider the coefficients $a_{0}=4 / 3, a_{1}=1 / 3$ with $r=2$. Then the sequence $\left\{V_{n}\right\}_{n=1}^{\infty}$ with $V_{n}=(-2 / 3)^{n}$ is an $r$-GFS associated with the coefficient sequence (1.3). If we put $V_{0}=-4 / 5, V_{-1}=6 / 5$, and $V_{n}=0$ for $n<-1$, then the sequence $\left\{V_{n}\right\}_{n \in \mathbb{Z}}$ is an $\infty$-GFS with respect to the coefficient sequence (2.2). Note that the sequence $\left\{(-2 / 3)^{n}\right\}_{n \in \mathbb{Z}}$ is not an $\infty$-GFS associated with the coefficient sequence (2.2) as pointed out in [2, Example 3.4]. 


\section{Strongly $\infty$-GFSs}

In this section, we introduce the notion of a strongly $\infty$-GFS and give a characterization theorem of periodic strongly $\infty$-GFSs. As a corollary, we give a Binet-type formula for such sequences.

Definition 3.1. A sequence $\left\{V_{n}\right\}_{n \in \mathbb{Z}}$ is called a strongly $\infty$-generalized Fibonacci sequence (strongly $\infty$-GFS) if (1.2) holds for all $n \in \mathbb{Z}$. Note that this notion already appeared in [7, Problem 3.11].

Let us consider an $r$-GFS $\left\{V_{n}\right\}_{n=1}^{\infty}$ associated with the coefficient sequence (1.3). Let $P(x)$ be the associated characteristic polynomial given by

$$
P(x)=x^{r}-a_{0} x^{r-1}-a_{1} x^{r-2}-\cdots-a_{r-2} x-\left(a_{r-1}+1\right) .
$$

For the moment, let us assume the condition

$$
a_{r-1} \neq-1 \text {. }
$$

We denote by $\lambda_{i}, 1 \leq i \leq k$, the roots of $P(x)$ with $\left|\lambda_{i}\right|>1$, and by $\lambda_{j}^{\prime}, 1 \leq j \leq \ell$, the roots of $P(x)$ with $0<\left|\lambda_{j}^{\prime}\right| \leq 1$. We assume that they are mutually distinct and denote by $m_{i} \geq 1$ and $m_{j}^{\prime} \geq 1$ the multiplicities of the roots $\lambda_{i}$ and $\lambda_{j}^{\prime}$, respectively. Note that

$$
\sum_{i=1}^{k} m_{i}+\sum_{j=1}^{\ell} m_{j}^{\prime}=r .
$$

Since $\left\{V_{n}\right\}_{n=1}^{\infty}$ is an $r$-GFS associated with the coefficient sequence (1.3) with $a_{r-1}+$ $1 \neq 0$, there exist complex numbers $\alpha_{i, s}$ and $\alpha_{j, t}^{\prime}$ such that

$$
V_{n}=\sum_{i=1}^{k} \sum_{s=0}^{m_{i}-1} \alpha_{i, s} n^{s} \lambda_{i}^{n}+\sum_{j=1}^{\ell} \sum_{t=0}^{m_{j}^{\prime}-1} \alpha_{j, t}^{\prime} n^{t}\left(\lambda_{j}^{\prime}\right)^{n}
$$

holds for all $n \geq 1$ by the Binet-type formula (for details see, e.g., [4]).

Then we have the following characterization theorem of those $r$-GFSs which can be extended to a periodic strongly $\infty$-GFS.

Theorem 3.2. Suppose that the condition (3.2) above holds. The sequence $\left\{V_{n}\right\}_{n=1}^{\infty}$ given by (3.4) can be extended to a strongly $\infty$-GFS $\left\{V_{n}\right\}_{n \in \mathbb{Z}}$ associated with the periodic coefficient sequence (2.2) if and only if $\alpha_{j, t}^{\prime}=0$ for all $j$ and all $t$.

Example 3.3. Let us consider the coefficient sequence as in Example 2.4. Then $\lambda_{1}=2$ and $\lambda_{2}=-2 / 3$ are the roots of the characteristic polynomial $P(x)$, which is of degree 2 . Then the sequence $\left\{2^{n}\right\}_{n \in \mathbb{Z}}$ is a periodic strongly $\infty$-GFS, while $\left\{(-2 / 3)^{n}\right\}_{n \in \mathbb{Z}}$ is not.

Remark 3.4. Consider the characteristic power series $Q(z)$ associated with the coefficient sequence $\left\{a_{i}\right\}_{i=0}^{\infty}$ defined by

$$
Q(z)=1-\sum_{i=0}^{\infty} a_{i} z^{i+1}
$$


(see $\left[1\right.$, Section 6]). If the sequence $\left\{a_{i}\right\}_{i=0}^{\infty}$ satisfies (2.1), then $Q(z)$ converges for $|z|<1$ and the equality

$$
Q\left(x^{-1}\right)=\frac{P(x)}{x^{r}-1}
$$

holds (see [2]). Furthermore, by [2], $\lambda_{i}$ are exactly the inverses of the zeros of the power series $Q(z)$ which lie inside the circle of convergence, and $m_{i}$ coincides with the order of the zero $\lambda_{i}^{-1}$.

Proof of Theorem 3.2. In the following, we use the notation $\left\{a_{i}\right\}_{i=0}^{\infty}$ as in the proof of Proposition 2.1.

Let us first assume that $\alpha_{j, t}^{\prime}=0$ for all $j$ and all $t$. In order to show that the sequence $\left\{V_{n}\right\}_{n \in \mathbb{Z}}$ given by (3.4) for all $n \in \mathbb{Z}$ is a strongly $\infty$-GFS associated with the periodic coefficient sequence (2.2), let us consider the sequence $\left\{W_{n}\right\}_{n \in \mathbb{Z}}$ with $W_{n}=n^{s} \lambda^{n}$, where $\lambda$ is a root of $P(x)$ with $|\lambda|>1$ with multiplicity $m$ and $0 \leq s \leq m-1$. Since it is an $r$-GFS associated with the coefficient sequence (1.3), for every $n \in \mathbb{Z}$ and $N>0$, we have

$$
\begin{aligned}
W_{n+1}= & a_{0} W_{n}+a_{1} W_{n-1}+\cdots+a_{r-2} W_{n-r+2}+\left(a_{r-1}+1\right) W_{n-r+1} \\
= & a_{0} W_{n}+a_{1} W_{n-1}+\cdots+a_{r-2} W_{n-r+2}+a_{r-1} W_{n-r+1}+W_{n-r+1} \\
= & a_{0} W_{n}+a_{1} W_{n-1}+\cdots+a_{r-2} W_{n-r+2}+a_{r-1} W_{n-r+1} \\
& +a_{0} W_{n-r}+a_{1} W_{n-r-1}+\cdots \\
& +a_{r-2} W_{n-2 r+2}+\left(a_{r-1}+1\right) W_{n-2 r+1} \\
= & \cdots=\sum_{i=0}^{N r-1} a_{i} W_{n-i}+W_{n-N r+1} .
\end{aligned}
$$

Note that

$$
\lim _{N \rightarrow \infty} W_{n-N r+1}=\lim _{N \rightarrow \infty}(n-N r+1)^{s} \lambda^{n-N r+1}=0
$$

holds, since $|\lambda|>1$ and $r>0$. Therefore, we have

$$
W_{n+1}=\sum_{i=0}^{\infty} a_{i} W_{n-i},
$$

where the series on the right-hand side converges. Hence the sequence $\left\{W_{n}\right\}_{n \in \mathbb{Z}}$ is a strongly $\infty$-GFS associated with the periodic coefficient sequence (2.2).

Therefore, if $\alpha_{j, t}^{\prime}=0$ for all $j$ and all $t$, then the sequence $\left\{V_{n}\right\}_{n \in \mathbb{Z}}$ given by (3.4) for all $n \in \mathbb{Z}$ is a strongly $\infty$-GFS associated with the periodic coefficient sequence (2.2).

Conversely, suppose that the sequence $\left\{V_{n}\right\}_{n=1}^{\infty}$ can be extended to a periodic strongly $\infty$-GFS $\left\{V_{n}\right\}_{n \in \mathbb{Z}}$ associated with the coefficient sequence (2.2). Let us first show that then $V_{n}$ should be given by (3.4) even for $n<0$. 
Let us fix an arbitrary negative integer $h$. First, note that the sequence $\left\{V_{n}\right\}_{n=h}^{\infty}$ is an $r$-GFS with respect to the coefficient sequence (1.3), since $\left\{V_{n}\right\}_{n \in \mathbb{Z}}$ is a strongly $\infty$-GFS. Therefore, there exist complex numbers $\beta_{i, s}$ and $\beta_{j, t}^{\prime}$ such that

$$
V_{n}=\sum_{i=1}^{k} \sum_{s=0}^{m_{i}-1} \beta_{i, s} n^{s} \lambda_{i}^{n}+\sum_{j=1}^{\ell} \sum_{t=0}^{m_{j}^{\prime}-1} \beta_{j, t}^{\prime} n^{t}\left(\lambda_{j}^{\prime}\right)^{n}
$$

holds for all $n \geq h$. Since the sequences $\left\{n^{s} \lambda_{i}^{n}\right\}_{n=0}^{\infty}\left(1 \leq i \leq k, 0 \leq s \leq m_{i}-1\right)$ and $\left\{n^{t}\left(\lambda_{j}^{\prime}\right)^{n}\right\}_{n=0}^{\infty}\left(1 \leq j \leq \ell, 0 \leq t \leq m_{j}^{\prime}-1\right)$ are linearly independent over the complex numbers, we see that $\beta_{i, s}=\alpha_{i, s}$ and $\beta_{j, t}^{\prime}=\alpha_{j, t}^{\prime}$ for all $i, s, j$, and $t$. Therefore, $V_{n}$ with $h \leq n<0$ should be given by (3.4). Since $h$ is an arbitrary negative integer, we see that every $V_{n}$ with $n<0$ should be given by (3.4).

We set

$$
A_{n}=\sum_{i=1}^{k} \sum_{s=0}^{m_{i}-1} \alpha_{i, s} n^{s} \lambda_{i}^{n}, \quad B_{n}=\sum_{j=1}^{\ell} \sum_{t=0}^{m_{j}^{\prime}-1} \alpha_{j, t}^{\prime} n^{t}\left(\lambda_{j}^{\prime}\right)^{n} .
$$

Since $V_{n}$ exists for all $n \in \mathbb{Z}$, the series

$$
\sum_{i=0}^{\infty} a_{i+n-1} V_{-i}=\sum_{i=1}^{\infty} a_{i+n-1}\left(A_{-i}+B_{-i}\right)
$$

converges for all $n \geq 1$ by [1]. It is easy to see that the series $\sum_{i=0}^{\infty} a_{i+n-1} A_{-i}$ converges (absolutely). Hence, the series $\sum_{i=0}^{\infty} a_{i+n-1} B_{-i}$ should also converge. In particular, we have $\lim _{i \rightarrow \infty} a_{i+n-1} B_{-i}=0$ for all $n \geq 1$. Since the coefficient sequence is periodic and $a_{i} \neq 0$ for some $a_{i}$, we should have $\lim _{i \rightarrow \infty} B_{-i}=0$.

Hence, it suffices to prove the following.

Lemma 3.5. Let $\lambda_{1}^{\prime}, \ldots, \lambda_{\ell}^{\prime}$ be distinct complex numbers such that $0<\left|\lambda_{j}^{\prime}\right| \leq 1$ for all $1 \leq j \leq$ $\ell$. Let $m_{j}^{\prime}$ be positive integers, $1 \leq j \leq \ell$. If

$$
\lim _{i \rightarrow \infty} \sum_{j=1}^{\ell}\left(\sum_{t=0}^{m_{j}^{\prime}-1} \alpha_{j, t}^{\prime}(-i)^{t}\right)\left(\lambda_{j}^{\prime}\right)^{-i}=0
$$

for complex numbers $\alpha_{j, t}^{\prime}$, then $\alpha_{j, t}^{\prime}=0$ for all $1 \leq j \leq \ell$ and all $0 \leq t \leq m_{j}^{\prime}-1$.

Proof. We will prove the lemma by induction on $\ell$. When $\ell=1$, we have

$$
B_{-i}=\left(\sum_{t=0}^{m_{1}^{\prime}-1} \alpha_{1, t}^{\prime}(-i)^{t}\right)\left(\lambda_{1}^{\prime}\right)^{-i}
$$

Suppose that $\alpha_{1, t}^{\prime} \neq 0$ for some $t$. Let $\tilde{t}$ be the largest $t$ with $\alpha_{1, t}^{\prime} \neq 0$. Then we have

$$
\lim _{i \rightarrow \infty}\left|\sum_{t=0}^{m_{1}^{\prime}-1} \alpha_{1, t}^{\prime}(-i)^{t}\right|= \begin{cases}+\infty & \text { if } \tilde{t}>0 \\ \left|\alpha_{1,0}^{\prime}\right|(\neq 0) & \text { if } \tilde{t}=0\end{cases}
$$


and hence we have $\lim _{i \rightarrow \infty}\left|B_{-i}\right|=+\infty$ or $\left|\alpha_{1,0}^{\prime}\right|$, since $\left|\lambda_{1}^{\prime}\right| \leq 1$. This is a contradiction. So, the assertion is valid for $\ell=1$.

Suppose now that $\ell \geq 2$ and that the assertion is true for $\ell-1$. We may assume that $\left|\lambda_{\ell}^{\prime}\right| \leq\left|\lambda_{j}^{\prime}\right|$ for all $1 \leq j \leq \ell-1$ and that $m_{\ell}^{\prime} \geq m_{j}^{\prime}$ for all $j$ with $\left|\lambda_{\ell}^{\prime}\right|=\left|\lambda_{j}^{\prime}\right|$. Since

$$
\lim _{i \rightarrow \infty} B_{-i}=\lim _{i \rightarrow \infty} \sum_{j=1}^{\ell}\left(\sum_{t=0}^{m_{j}^{\prime}-1} \alpha_{j, t}^{\prime}(-i)^{t}\right)\left(\lambda_{j}^{\prime}\right)^{-i}=0,
$$

we have

$$
\begin{aligned}
0 & =\lim _{i \rightarrow \infty} \frac{1}{(-i)^{m_{\ell}^{\prime}-1}} \sum_{j=1}^{\ell}\left(\sum_{t=0}^{m_{j}^{\prime}-1} \alpha_{j, t}^{\prime}(-i)^{t}\right)\left(\frac{\lambda_{\ell}^{\prime}}{\lambda_{j}^{\prime}}\right)^{i} \\
& =\lim _{i \rightarrow \infty}\left[\sum_{j=1}^{\ell-1}\left(\sum_{t=0}^{m_{j}^{\prime}-1} \alpha_{j, t}^{\prime}(-i)^{t-m_{\ell}^{\prime}+1}\right)\left(\frac{\lambda_{\ell}^{\prime}}{\lambda_{j}^{\prime}}\right)^{i}+\left(\sum_{t=0}^{m_{\ell}^{\prime}-1} \alpha_{\ell, t}^{\prime}(-i)^{t-m_{\ell}^{\prime}+1}\right)\right] .
\end{aligned}
$$

Set

$$
\Lambda=\left\{1 \leq j \leq \ell-1:\left|\lambda_{j}^{\prime}\right|=\left|\lambda_{\ell}^{\prime}\right|\right\}, \quad \tilde{\Lambda}=\left\{j \in \Lambda: m_{j}^{\prime}=m_{\ell}^{\prime}\right\} .
$$

Note that for $j$ with $\left|\lambda_{\ell}^{\prime}\right|<\left|\lambda_{j}^{\prime}\right|$, we have

$$
\lim _{i \rightarrow \infty}\left(\sum_{t=0}^{m_{j}^{\prime}-1} \alpha_{j, t}^{\prime}(-i)^{t-m_{\ell}^{\prime}+1}\right)\left(\frac{\lambda_{\ell}^{\prime}}{\lambda_{j}^{\prime}}\right)^{i}=0 .
$$

Therefore, we obtain

$$
\lim _{i \rightarrow \infty}\left[\sum_{j \in \Lambda}\left(\sum_{t=0}^{m_{j}^{\prime}-1} \alpha_{j, t}^{\prime}(-i)^{t-m_{\ell}^{\prime}+1}\right)\left(\frac{\lambda_{\ell}^{\prime}}{\lambda_{j}^{\prime}}\right)^{i}+\left(\sum_{t=0}^{m_{\ell}^{\prime}-1} \alpha_{\ell, t}^{\prime}(-i)^{t-m_{\ell}^{\prime}+1}\right)\right]=0 .
$$

Furthermore, we have

$$
\lim _{i \rightarrow \infty} \sum_{t=0}^{m_{\ell}^{\prime}-1} \alpha_{\ell, t}^{\prime}(-i)^{t-m_{\ell}^{\prime}+1}=\alpha_{\ell, m_{\ell}^{\prime}-1}^{\prime}
$$

and for all $j \in \Lambda-\tilde{\Lambda}$, we have

$$
\lim _{i \rightarrow \infty} \sum_{t=0}^{m_{j}^{\prime}-1} \alpha_{j, t}^{\prime}(-i)^{t-m_{\ell}^{\prime}+1}=0
$$

Therefore, we obtain

$$
\lim _{i \rightarrow \infty} \sum_{j \in \tilde{\Lambda}}\left(\sum_{t=0}^{m_{\ell}^{\prime}-1} \alpha_{j, t}^{\prime}(-i)^{t-m_{\ell}^{\prime}+1}\right)\left(\frac{\lambda_{\ell}^{\prime}}{\lambda_{j}^{\prime}}\right)^{i}=\lim _{i \rightarrow \infty} \sum_{j \in \tilde{\Lambda}} \alpha_{j, m_{\ell}^{\prime}-1}^{\prime}\left(\frac{\lambda_{\ell}^{\prime}}{\lambda_{j}^{\prime}}\right)^{i}=-\alpha_{\ell, m_{\ell}^{\prime}-1}^{\prime} .
$$


If we set

$$
b_{i}=\sum_{j \in \widetilde{\Lambda}} \alpha_{j, m_{\ell}^{\prime}-1}^{\prime}\left(\frac{\lambda_{\ell}^{\prime}}{\lambda_{j}^{\prime}}\right)^{i}
$$

then this implies that

$$
\lim _{i \rightarrow \infty}\left(b_{i+1}-b_{i}\right)=\lim _{i \rightarrow \infty} \sum_{j \in \tilde{\Lambda}} \alpha_{j, m_{\ell}^{\prime}-1}^{\prime}\left(\frac{\lambda_{\ell}^{\prime}}{\lambda_{j}^{\prime}}-1\right)\left(\frac{\lambda_{\ell}^{\prime}}{\lambda_{j}^{\prime}}\right)^{i}=0,
$$

and hence that

$$
\lim _{i \rightarrow \infty} \sum_{j \in \tilde{\Lambda}} \alpha_{j, m_{\ell}^{\prime}-1}^{\prime}\left(\frac{\lambda_{\ell}^{\prime}}{\lambda_{j}^{\prime}}\right)^{i}=0
$$

Note that $\left|\lambda_{\ell}^{\prime} / \lambda_{j}^{\prime}\right|=1$. Since the number of elements of $\tilde{\Lambda}$ is strictly smaller than $\ell$, we have, by our induction hypothesis, that $\alpha_{j, m_{\ell}^{\prime}-1}^{\prime}=0$ for all $j \in \tilde{\Lambda}$.

Repeating this procedure finitely many times, we can finally show that $\alpha_{j, t}^{\prime}=0$ for all $1 \leq j \leq \ell$ and all $0 \leq t \leq m_{j}-1$. This completes the proof of Lemma 3.5.

This completes the proof of Theorem 3.2.

Remark 3.6. If the condition (3.2) is not satisfied, then $V_{n}, n \geq 1$, may not be given by (3.4). More precisely, let $r^{\prime}$ be the largest integer with $r^{\prime}<r$ such that $a_{r^{\prime}-1} \neq 0$, and set $u=r-r^{\prime}+1$. If such an $a_{r^{\prime}-1}$ does not exist, then set $r^{\prime}=0$. Then the sequence $\left\{V_{n}\right\}_{n=u}^{\infty}$ is an $r^{\prime}$-GFS, and the terms $V_{n}$ with $1 \leq n<u$ may not satisfy (3.4), where a "0-GFS" conventionally means the sequence that is constantly zero.

As a corollary to Theorem 3.2, we have a Binet-type formula for periodic strongly $\infty$ GFSs as follows, where we do not assume the condition (3.2) any more.

Corollary 3.7. Let $\left\{V_{n}\right\}_{n \in \mathbb{Z}}$ be a periodic strongly $\infty$-GFS associated with the periodic coefficient sequence (2.2). Then

$$
V_{n}=\sum_{i=1}^{k} \sum_{s=0}^{m_{i}-1} \alpha_{i, s} n^{s} \lambda_{i}^{n}
$$

for all $n \in \mathbb{Z}$ for some complex numbers $\alpha_{i, s}$, where $\lambda_{i}$ are the inverses of the zeros of the characteristic power series given by (3.5) and satisfy $\left|\lambda_{i}\right|>1$.

In other words, the roots of $P(x)$ whose moduli are less than or equal to 1 do not appear in the formula. In view of Remark 3.4, Corollary 3.7 gives a positive solution to [7, Problem 4.5] for periodic strongly $\infty$-GFSs. In order to get a Binet-type formula, we should not take the zeros of an analytic continuation of $Q(z)$, but take the zeros of $Q(z)$ inside the circle of convergence.

Proof of Corollary 3.7. If the condition (3.2) is satisfied, then the conclusion follows immediately from Theorem 3.2. 
Suppose that the condition (3.2) is not satisfied. Take the integer $r^{\prime}$ as in Remark 3.6. Let us first assume that $r^{\prime}>0$. Since $\left\{V_{n}\right\}_{n \in \mathbb{Z}}$ is a periodic strongly $\infty$-GFS associated with the coefficient sequence (2.2), the sequence $\left\{V_{n}\right\}_{n=h}^{\infty}$ is an $r^{\prime}$-GFS associated with the coefficient sequence

$$
\left\{a_{0}, a_{1}, \ldots, a_{r^{\prime}-1}\right\}
$$

for any $h \in \mathbb{Z}$ by [2]. Therefore, $V_{n}, n \geq 1$, can be expressed as in (3.4), where $\lambda_{i}$ and $\lambda_{j}^{\prime}$ are the roots of the characteristic polynomial associated with the truncated coefficient sequence (3.28). Then the argument in the proof of Theorem 3.2 can be applied to prove the desired conclusion.

If $r^{\prime}=0$, then $a_{0}=a_{1}=\cdots=a_{r-2}=0$ and $a_{r-1}=-1$. In this case, the sequence $\left\{V_{n}\right\}_{n \in \mathbb{Z}}$ is easily seen to be constantly zero. Hence the conclusion trivially holds. This completes the proof.

In fact, we have the following characterization of strongly $\infty$-GFSs associated with a periodic coefficient sequence, which follows from the proof of Theorem 3.2 together with Corollary 3.7.

Corollary 3.8. A sequence $\left\{V_{n}\right\}_{n \in \mathbb{Z}}$ is a strongly $\infty$-GFS associated with the periodic coefficient sequence (2.2) if and only if

$$
V_{n}=\sum_{i=1}^{k} \sum_{s=0}^{m_{i}-1} \alpha_{i, s} n^{s} \lambda_{i}^{n}
$$

holds for all $n \in \mathbb{Z}$ for some complex numbers $\alpha_{i, s}$, where $\lambda_{i}$ are the inverses of the zeros of the characteristic power series given by (3.5) and satisfy $\left|\lambda_{i}\right|>1$.

Note that Corollary 3.8 gives a complete solution to [7, Problem 3.11] in the case where the coefficient sequence is periodic.

Remark 3.9. As has been observed in [2, Remark 2.5], the subsequence $\left\{V_{n}\right\}_{n=1}^{\infty}$ of a periodic $\infty$-GFS $\left\{V_{n}\right\}_{n \in \mathbb{Z}}$ can be considered as a $k r$-GFS with respect to the coefficient sequence $\left\{a_{0}, \ldots, a_{k r-2}, a_{k r-1}+1\right\}$, where $k$ is an arbitrary positive integer. Let $P^{(k)}$ be the associated characteristic polynomial. Then we have

$$
\begin{aligned}
P^{(k)}(x) & =x^{k r}-a_{0} x^{k r-1}-\cdots-a_{k r-2} x-\left(a_{k r-1}+1\right) \\
& =x^{k r}-1-\left(a_{0} x^{r-1}+\cdots+a_{r-1}\right) \frac{x^{k r}-1}{x^{r}-1} \\
& =\frac{x^{k r}-1}{x^{r}-1} P(x) .
\end{aligned}
$$

Thus the roots of $P=P^{(1)}$ are also roots of $P^{(k)}$. The other roots of $P^{(k)}$ are all $k r$ th roots of unity and these roots do not appear in the Binet-type formula according to Corollary 3.7.

Let us end this section by posing a problem, which is closely related to [7, Problem 3.11]. 
Problem 3.10. Suppose that a sequence $\left\{V_{n}\right\}_{n=1}^{\infty}$ can be extended to an $\infty$-GFS. Then, can it be extended to a strongly $\infty$-GFS? If yes, then is such an extension unique?

Remark 3.11. We can also define a strongly $r$-GFS, imitating Definition 3.1. As we can easily show, if the coefficient sequence $\left\{a_{i}\right\}_{i=0}^{r-1}$ satisfies $a_{r-1} \neq 0$, then every $r$-GFS $\left\{V_{n}\right\}_{n=1}^{\infty}$ can be extended uniquely to a strongly $r$-GFS associated with the same coefficient sequence.

\section{Acknowledgments}

The second author has been partially supported by Grant-in-Aid for Scientific Research (no. 16340018), Japan Society for the Promotion of Science. He was also partially supported by the Louis Pasteur University of Strasbourg, France. He would like to express his thanks to the people at the Louis Pasteur University of Strasbourg for their hospitality during the preparation of the manuscript. The authors would like to express their thanks to Professors B. Bernoussi and W. Motta for useful discussions. They also would like to thank the referees for many helpful comments.

\section{References}

[1] B. Bernoussi, W. Motta, M. Rachidi, and O. Saeki, Approximation of $\infty$-generalized Fibonacci sequences and their asymptotic Binet formula, The Fibonacci Quarterly 39 (2001), no. 2, 168 180.

[2] _ On periodic $\infty$-generalized Fibonacci sequences, The Fibonacci Quarterly 42 (2004), no. 4, 361-367.

[3] F. Dubeau, On r-generalized Fibonacci numbers, The Fibonacci Quarterly 27 (1989), no. 3, $221-$ 229.

[4] F. Dubeau, W. Motta, M. Rachidi, and O. Saeki, On weighted r-generalized Fibonacci sequences, The Fibonacci Quarterly 35 (1997), no. 2, 102-110.

[5] C. Levesque, On m-th order linear recurrences, The Fibonacci Quarterly 23 (1985), no. 4, 290293.

[6] E. P. Miles Jr., Generalized Fibonacci numbers and associated matrices, The American Mathematical Monthly 67 (1960), no. 8, 745-752.

[7] W. Motta, M. Rachidi, and O. Saeki, On $\infty$-generalized Fibonacci sequences, The Fibonacci Quarterly 37 (1999), no. 3, 223-232.

[8] _ Convergent $\infty$-generalized Fibonacci sequences, The Fibonacci Quarterly 38 (2000), no. $4,326-333$.

[9] M. Mouline and M. Rachidi, Suites de Fibonacci généralisées et chaînes de Markov, Real Academia de Ciencias Exactas, Físicas y Naturales de Madrid. Revista 89 (1995), no. 1-2, 61-77.

[10] _,$\infty$-Generalized Fibonacci sequences and Markov chains, The Fibonacci Quarterly 38 (2000), no. 4, 364-371.

Mustapha Rachidi: Section de Mathématique, LEGT - F. Arago, Académie de Reims, 1, rue F. Arago, Reims 51100, France

E-mail address: mu.rachidi@wanadoo.fr

Osamu Saeki: Faculty of Mathematics, Kyushu University, Hakozaki, Fukuoka 812-8581, Japan

E-mail address: saeki@math.kyushu-u.ac.jp 